\title{
RNA interference against granulin-epithelin precursor prevents hepatocellular carcinoma growth: Its application as a therapeutic agent
}

\author{
MI-YOUNG PARK ${ }^{1 *}$, YOON SHIN PARK ${ }^{2 *}$ and JAE-HWAN NAM ${ }^{1}$ \\ ${ }^{1}$ Department of Biotechnology, The Catholic University of Korea, Bucheon 420-743, Republic of Korea; \\ ${ }^{2}$ Department of Pharmeceutical Sciences, College of Pharmacy, The University of Michigan, \\ 428 Church Street, Ann Arbor, MI 48109-1065, USA
}

Received March 22, 2011; Accepted May 2, 2011

DOI: 10.3892/ijo.2011.1095

\begin{abstract}
Hepatocellular carcinoma (HCC) is the fifth most common cancer in the world. However, little is known regarding the molecular mechanism of HCC development and progression and effective therapeutic methods. Recently, the granulinepithelin precursor (GEP) was reported as a novel growth factor that can control HCC cell proliferation. Using the CAPSID program, we designed three small interfering RNAs (siRNAs) targeting the GEP gene (GEP-siRNA1, 2 and 3) and examined their tumor regression and suppression effects on cell proliferation. GEP-siRNA1 exhibited the strongest anti-proliferative effect among the GEP-siRNAs, in a time-dependent manner. To increase the biostability of the siRNA, we also constructed a short hairpin RNA (shRNA) using an H1/TO promoter with the same sequence of GEP-siRNA1 (GEP-shRNA). GEP-shRNA decreased the expression levels of GEP and tumor cell growth via cell cycle arrest at the G2/M stage and down-regulation of the cell proliferation proteins cyclin D1 and $\alpha$-tubulin. Furthermore, GEP-shRNA inhibited tumor growth significantly after intratumoral injection into tumor-bearing $\mathrm{Balb} / \mathrm{C}$ nude mice. Taken together, these results represent the first therapeutic application of RNA interference to GEP, which is a promising target molecule for HCC treatment, as an approach for the suppression of HCC cell proliferation.
\end{abstract}

Correspondence to: Dr Jae-Hwan Nam, Division of Biotechnology, The Catholic University of Korea, 43-1 Yeokgok 2-dong, Wonmi-gu, Gyeonggi-do, Bucheon, Republic of Korea

E-mail: jhnam@catholic.ac.kr

${ }^{*}$ Contributed equally

Abbreviations: HCC, hepatocellular carcinoma; GEP, granulinepithelin precursor; siRNA, small interfering RNA; shRNA, short hairpin RNA; RNAi, RNA interference; $\mathrm{HCV}$, hepatitis $\mathrm{C}$ virus; $\mathrm{HBV}$, hepatitis B virus; PCDGF, PC cell-derived growth factor; GEM, granulin-epithelin module; CAPSID, convenient application program for siRNA design

Key words: HCC, granulin-epithelin precursor, siRNA, shRNA, cell proliferation protein

\section{Introduction}

Hepatocellular carcinoma (HCC) is the most common primary malignant liver cancer in adults and is a highly prevalent disease worldwide, with most patients presenting a highly advanced disease status and thus poor prognosis (1). HCC is also known as the third most common cause of cancer-related deaths among patients with cirrhosis in Europe and the USA (2-5). The most relevant risk factor for HCC is liver injury from diverse causes, which induces hepatic cirrhosis in most patients; these causes include chronic hepatitis C (HCV) infection, hepatitis B (HBV) infection, alcoholic cirrhosis, and nonalcoholic steatohepatitis (6). Furthermore, the increasing incidence of obesity and diabetes, which was also identified as an independent risk factor for chronic liver disease and HCC, is becoming a major issue in the treatment of HCC (7). Despite advances in many aspects of $\mathrm{HCC}$ treatment, including liver transplantation and surgical resection, over two-thirds of HCC patients present with advanced disease and do not benefit from these treatment modalities (7). To date, surgical liver transplantation is considered the only curative treatment for HCC and only one chemotherapeutic agent is approved by the United States Food and Drug Administration for the treatment of advanced HCC (8). However, most HCC patients are not candidates for liver transplantation or surgical resection because of their advanced disease status (9) and the majority of HCC patients represents a significant medical need for more effective systemic therapy options. Furthermore, as HCC tumor tissues have high chemoresistance, systemic chemotherapy is not a promising treatment. In addition, the molecular pathogenesis of HCC remains poorly understood and its clinical course varies widely (10). Thus, many recent investigations have indicated that molecularly targeted therapies are an encouraging trend in the management of $\operatorname{HCC}(10,11)$.

The granulin-epithelin precursor (GEP), also termed progranulin, acrogranin, and PC-derived growth factor (PCDGF), is a 68.5-kDa pluripotent glycoprotein and an obscure cysteine-rich novel growth factor (12). GEP is structurally composed of seven and one-half granulin/epithelin module (GEM) repeats (12). The GEMs are separated by a 6-kDa peptide fragment and are made up of four $\beta$ hairpins stacked onto each other in a twisted ladder- 
like formation (13). Generally, GEP affects many biological features, such as development, cell growth and proliferation, host defense, and wound healing (14). In addition, overexpression of GEP is associated with cancer progression (15). Strong GEP expression is highly associated with HCC growth with a large tumor size and GEP is considered as an important factor in hepatocarcinogenesis and a promising factor that can be used as a tumor marker or as a therapeutic target molecule (15).

After the first report of strong GEP expression from HCC tissues, antisense GEP and a monoclonal antibody to GEP were used to block the expression of GEP in an HCC mouse model $(15,16)$, which demonstrated that GEP agents can be used as safe anti-HCC therapies that delay tumor growth. However, the effect of antisense GEP was not so effective in a highly overexpressed GEP system, which suggests that this mechanism is only effective for intermediate-to-low levels of GEP expression $(15,16)$.

Recently, the examination of RNA interference (RNAi) using small interfering RNA (siRNA) and short hairpin RNA (shRNA) in a preclinical cancer study yielded promising results, including an efficient inhibitory effect on tumor angiogenesis, tumor cell growth, tumor invasion, and chemoresistance $(17,18)$. Therefore, we designed three different types of siRNAs (GEP-siRNAs) and one shRNA (GEP-shRNA) against GEP using the CAPSID (Convenient Application Program for siRNA Design) software. The cell-growth inhibitory effect of the siRNA and shRNA molecules constructed in this study was tested on HCC. They induced the inhibition of tumor-cell proliferation and a decrease in tumor size via cell-cycle arrest at the G2/M stage. In conclusion, GEP-siRNA and GEP-shRNA seem to be effective therapeutic agents for HCC.

\section{Materials and methods}

Cell culture and GEP cloning. The HCC cell lines Hep3B, Huh7, and skHep1 were cultured in Dulbecco's modified Eagle's medium (Hyclone, Rockford, IL, USA) supplemented with heat-inactivated $10 \%$ fetal bovine serum (Hyclone), $50 \mathrm{U} / \mathrm{ml}$ penicillin, and $50 \mu \mathrm{g} / \mathrm{ml}$ streptomycin (Invitrogen, Carlsbad, CA, USA). Cells were maintained at $37^{\circ} \mathrm{C}$ in a humidified chamber with $5 \% \mathrm{CO}_{2}$. For the cloning of $G E P$, the full-length GEP cDNA cloned in pAcGP67-A (provided by the 21C Frontier Human Gene Bank, Daejeon, Republic of Korea) was used as a template for the fusion of $G E P$ and human $\operatorname{Ig} G F C$ (which was used as a tag) into pcDNA3.1(+) (pcDNA-FC-GEP) (Invitrogen). The pcDNA-FC-GEP was obtained from the subcloning of fulllength GEP using HindW and XhoI enzymatic digestion. The detected plasmid clone is shown in Fig. 1.

Design of siRNAs and shRNA. The cDNA sequence of GEP was obtained from GenBank (access number BC010577). Three short interfering RNAs (siRNAs) targeting GEP, GEP-siRNA1 (5'-AAGGACACUUCUGCCAUGAUAAC-3'), GEP-siRNA2 (5'-AAGAGAGAUGUCCCCUGUGAUAA-3'), and GEPsiRNA3 (5'-AAUCUAAGGCCUUCCCUGUCAGA-3'), were designed using the CAPSID program (http://cms.ulsan.ac.kr/ capsid). The HCC cell lines were used to examine the interfering effect of the three siRNA duplexes. GEP-shRNA containing GEP-siRNA1 sequences was constructed using an H1/TO promoter. A luciferase-gene-specific shRNA (LuciferaseshRNA) obtained from Genolution Pharmaceuticals Inc. (Seoul,

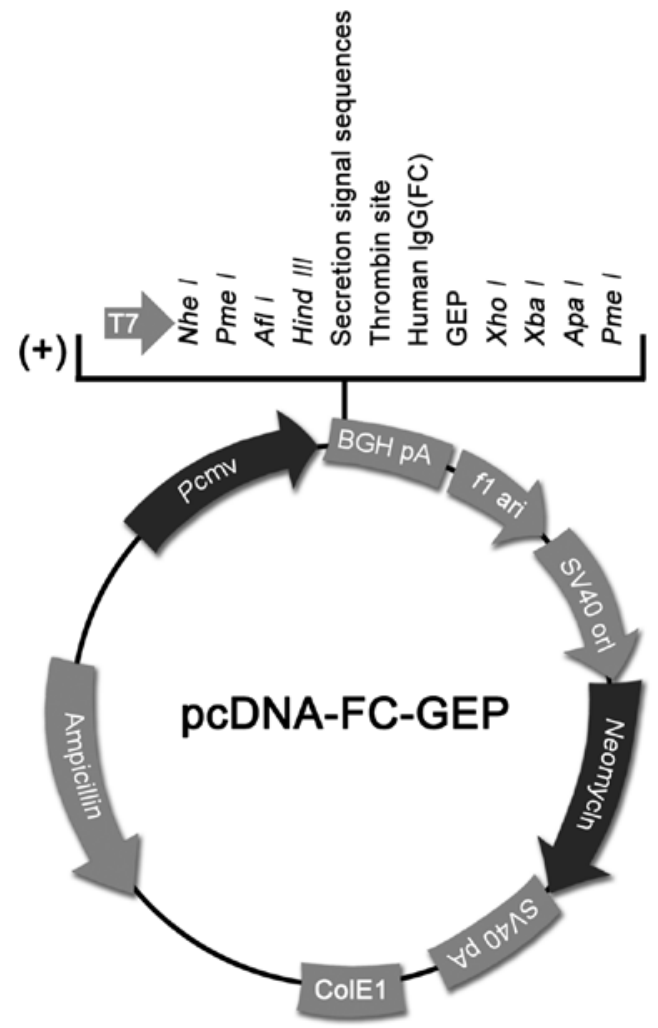

Figure 1. Construction of pcDNA-FC-GEP.

Republic of Korea) was used as a negative control. Transfection of the GEP-siRNAs and GEP-shRNA was performed using a Lipofectamine 2000 (Invitrogen) according to the manufacturer's instructions.

Human IgG ELISA. The level of human IgG FC expression measured by an ELISA kit (Koma Biotech, Seoul, Republic of Korea) was used to monitor the GEP expression in the supernatants of Hep3B cells transfected with pcDNA-FC-GEP.

Cell proliferation and viability assay. A cell proliferation assay was performed to count viable cells using a hemacytometer. HCC cells grown in 24 -well plates $\left(1 \times 10^{4}\right.$ cells/well) were transfected with $2 \mu \mathrm{g}$ of GEP-shRNA or Luciferase-shRNA using Lipofectamine 2000. Cells were harvested 1,3, and 5 days after transfection and viable cells were counted by trypan blue exclusion and counted using a hemacytometer. In addition, cell viability was assessed by measuring the optical density at $490 \mathrm{~nm}$ using the CellTiter $96^{\circledR}$ AQueous One Solution Cell Proliferation Assay kit (Promega, Madison, WI, USA) according to the manufacturer's protocol.

Real-time RT-PCR. Total RNA was extracted from Hep3B cells using TRIzol (Invitrogen). Total RNA samples ( $2 \mu \mathrm{g})$ were used in real-time RT-PCR experiments. First-strand cDNA was synthesized using Oligo-dT and RT\&GO ${ }^{\mathrm{TM}}$ (Q-Biogene Inc., Montreal, Canada) according to the manufacturer's instructions. The primer pairs used for the amplification of specific cDNAs were as follows: GEP, 5'-TCCACGTGCTGTGTTATGGT-3' (sense) and 5'-CTGCCCTGTTAGTCCTCTGG-3' (antisense); 18S rRNA, 5'-AACTTTCGATGGTAGTCGCCG-3' (sense) 
and 5'-GGATGTGGTAGCCGTTTCTCA-3' (antisense). Realtime RT-PCR amplification mixtures $(25 \mu \mathrm{l})$ contained 500 ng of template cDNA, $12.5 \mu \mathrm{l}$ of SYBR Green I Master Mix buffer (Applied Biosystems, Foster City, CA, USA), and $1 \mu \mathrm{l}$ of forward and reverse primers. The MyiQ Single-Color Real-time PCR Detection System (Bio-Rad, Hercules, CA, USA) was used to perform real-time quantitative RT-PCR.

Tumorigenicity assay using tumor-bearing Balb/C nude mice. Male Balb/C mice (4 weeks old) were obtained from Orient Bio Inc. (Seongnam, Republic of Korea), housed in special pathogenfree units, and maintained in the animal facility at the Catholic University according to the institutional guidelines. An in vivo tumorigenesis assay performed to assess the effect of the transfectants was modified from the previously reported method $(19,20)$. In brief, Hep3B cells $\left(1 \times 10^{7}\right.$ cells) were injected subcutaneously into the femoral region of mice. Tumor formation was confirmed after 4 weeks of injection. All tumor-bearing mice were divided into two groups, one injected with GEP-shRNA and the other with Luciferase-shRNA (which was used as a negativecontrol group). Mice were administered intratumoral injections of $30 \mu \mathrm{g}$ of GEP-shRNA in $30 \mu \mathrm{l}$ of PBS or $30 \mu \mathrm{g}$ of LuciferaseshRNA in $30 \mu \mathrm{l}$ of PBS every 4 days. Tumor dimensions after transfection were measured every day for 31 days using a digital caliper and tumor volume was calculated using the formula $\mathrm{V}=\pi / 6 \mathrm{x}$ larger diameter $\mathrm{x}$ smaller diameter $(2,19,20)$.

Western blot assay. Total protein was extracted from transfected Hep3B cells using M-PER buffer (Pierce, Rockford, IL, USA) containing Protease Inhibitor Cocktail ${ }^{\mathrm{TM}}$ (Roche Molecular Biochemicals, Indianapolis, IN, USA). Protein concentrations were determined using the BCA protein assay kit (Sigma-Aldrich Corp., St. Louis, MO, USA). Equal quantities of protein (30 $\mu \mathrm{g})$ were separated using sodium dodecyl sulfate-polyacrylamide gel electrophoresis (SDS-PAGE) under reducing conditions and were electrophoretically transferred onto a nitrocellulose membrane. The blots were probed with the appropriate antibodies against GEP (1:2,000, Santa Cruz Biotechnology, Santa Cruz, CA, USA), cyclin D1 (1:2,000, Santa Cruz Biotechnology), $\alpha$-tubulin (1:4,000, Abfrontier, Seoul, Republic of Korea), and $\beta$-actin $(1: 4,000$, Cell Signaling Technology, Danvers, MA, USA), followed by incubation with the corresponding secondary antibodies, and were developed using an ECL solution (Amersham Biosciences, Piscataway, NJ, USA). The level of $\beta$-actin expression was used as an internal control. The band densities were quantified using the ImageJ program (National Institute of Health, Bethesda, MD, USA).

Cell cycle analysis. Cell cycle analysis was performed using a flow cytometer. Hep3B cells were transfected with GEP-shRNA and Luciferase-shRNA using Lipofectamine 2000. Five days after transfection, cultures were trypsinized and centrifuged to collect the cells. Collected cells were then stained using propidium iodide ( $40 \mu \mathrm{g} / \mathrm{ml}$; Sigma) in PBS after washing twice with PBS. Cell cycle data were collected using a Beckman Coulter FC500 instrument (Beckman Coulter Inc., Brea, CA, USA).

Statistical analysis. The significance of differences between values was determined using the Student's t-test with the SAS 9.1 software (SAS Institute Inc., Cary, NC, USA). All data are
A

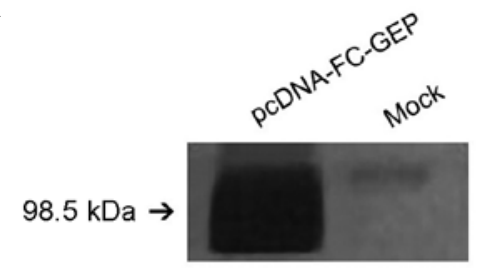

B

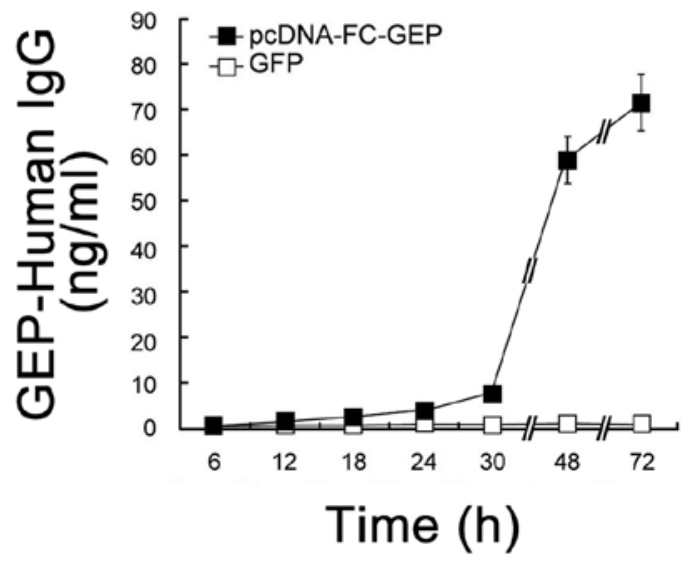

Figure 2. Expression of FC-tagged GEP in Hep3B cells. (A), Western blot analysis using an anti-GEP antibody to detect the $98.5 \mathrm{kDa}$ band of the FC-tagged GEP protein in pcDNA-FC-GEP-transfected Hep3B cells. Non-transfected Hep3B cells were used as a mock. (B), The level of secreted FC in pcDNA-FCGEP-transfected Hep3B culture medium, which is representative of the level of GEP expression because FC was fused to GEP, was detected using ELISA. GFP-transfected cells (GFP) were used as a control group

expressed as the mean \pm SD of triplicates. p-values $<0.05$ were considered statistically significant.

\section{Results}

Detection of FC-tagged GEP secretion. Transfection of $2 \mu \mathrm{g}$ of pcDNA-FC-GEP into Hep3B cells using Lipofectamine 2000 led to the expression of a 98.5-kDa FC-GEP protein compared with non-GEP-transfected Hep3B cells (mock) (Fig. 2A). We also confirmed the secretion of GEP in the supernatant of pcDNAFC-GEP-transfected Hep3B cells using an ELISA, which can detect the amount of FC fused with GEP. The expression of the GEP protein increased gradually after $24 \mathrm{~h}$ of transfection (up to $72 \mathrm{~h}$ ), with a dramatic increase at $48 \mathrm{~h}$ after pcDNA-FC-GEP transfection compared with in GFP-transfected Hep3B cells (Fig. 2B)

Design of three GEP-siRNAs and their inhibitory effect on GEP expression in Hep3B cells. Three structurally different GEP-siRNAs were designed using the CAPSID program and were used to check the applicability of RNAi as a therapeutic agent for HCC. The three regions of the GEP-siRNAs were designed using GEP sequences encoding stem-loop structures, which suggest the presence of biologically active regions (Fig. 3A). We compared the GEP-suppression effect of all three GEP-siRNAs to select the most effective RNA interfering sequence. The three GEP-siRNAs yielded a similar GEP-suppression effect at 1 day after transfection. However, 
A

GEP-siRNA1

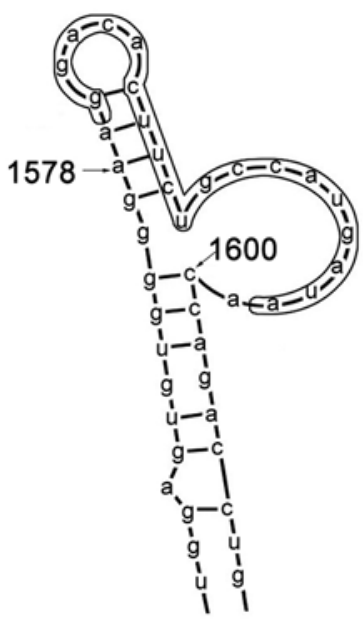

GEP-siRNA2

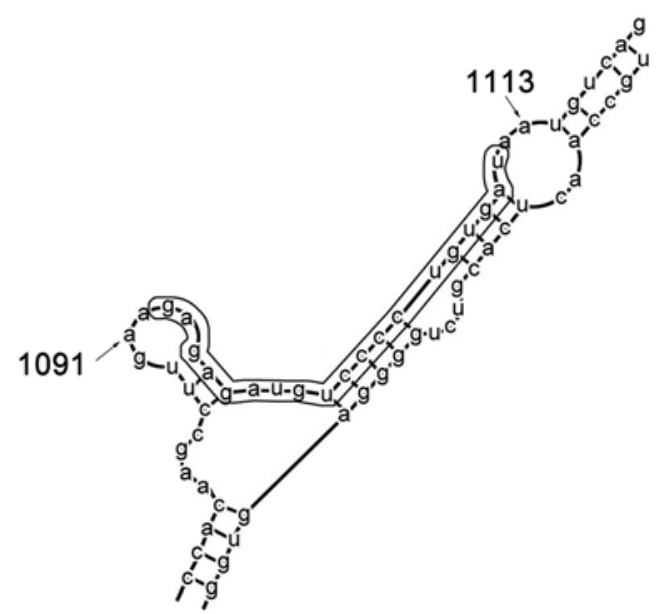

\section{GEP-SiRNA3}

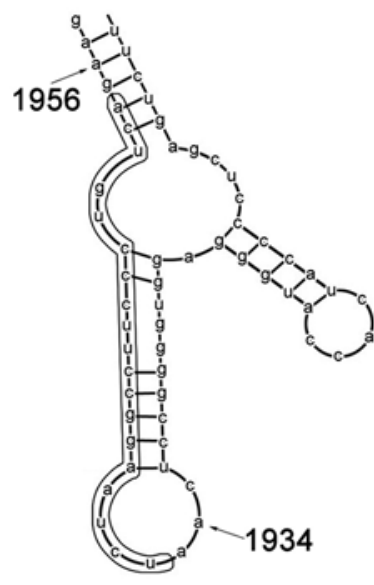

B

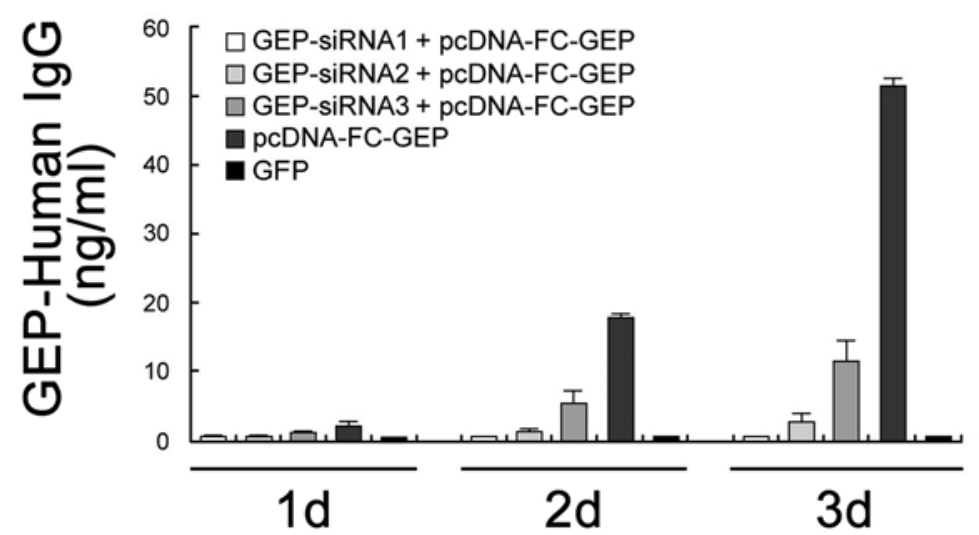

Figure 3. Design of GEP small interference RNAs (GEP-siRNAs) using the CAPSID program and their inhibitory effect on GEP expression in GEP-siRNAtransfected Hep3B cells. (A), Three different structures of GEP-siRNAs designed using CAPSID. The box in stem-loop structures indicates the sequences selected for the siRNAs against GEP. (B), GEP-siRNAs suppressed GEP secretion, as measured using ELISA. GEP-siRNA1 yielded the most significant GEP-suppression effect in Hep3B cells, in a time-dependent manner, compared with GEP-siRNA2 and GEP-siRNA3. All groups were cotransfected with pcDNA-FC-GEP ( $2 \mu$ g) and GEP-siRNAs (100 pmol). GFP transfection was used as a negative control.

compared with GEP-siRNA1, GEP-siRNA2 and GEP-siRNA3 exhibited a weak GEP-suppression effect 2 and 3 days after transfection, respectively (Fig. 3B). These results led us to select GEP-siRNA1 as the most effective GEP-suppressing agent among the three siRNAs.

GEP-shRNA effectively suppressed GEP expression in Hep3B cells. To increase the stability and handling efficiency of the agent for the application both in in vitro and in vivo experiments, an shRNA plasmid containing the GEP-siRNA1 sequence (termed GEP-shRNA) was constructed. To investigate the GEP-silencing effect of GEP-shRNA in Hep3B cells, four different components, GEP-shRNA, GEP-siRNA1, pcDNA-FC-GEP, and GFP (which was used as a mock), were transfected into Hep3B cells, individually. Using an ELISA assay to detect FC levels secreted to the cell culture medium, we confirmed the presence of a GEP-suppression effect in GEP-shRNA-transfected cells that was similar to that observed in GEP-siRNA1-transfected cells (Fig. 4A). In addition, over $0.5 \mu \mathrm{g}$ of GEP-shRNA was sufficient to achieve suppression of GEP expression (Fig. 4B). Moreover, GEP-shRNA down-regulated the level of the endogenous GEP mRNA in Hep3B cells by $74 \%(74 \pm 4 \%, \mathrm{p}<0.05)$ (Fig. 4C).

GEP-shRNA-mediated growth inhibition and cell cycle arrest in Hep3B cells. To test the cell growth-inhibition effect of GEP-shRNA, we measured cell proliferation using a hemacytometer and analyzed cell viability using a commercially available kit to detect the amount of viable GEP-shRNAtransfected cells in three different HCC cell lines (Hep3B, Huh7, and skHep1 cells). At 1, 3, and 5 days after transfection, the number of GEP-shRNA-transfected cells counted using trypan blue staining was significantly decreased compared with pcDNA-FC-GEP-transfected cells ( $\mathrm{p}<0.01$ in Hep3B, $\mathrm{p}<0.001$ in Huh7, and $\mathrm{p}<0.01$ in skHep1 cells) (Fig. 5A). Moreover, a cell viability determined by an OD values yielded similar results with the cell-counting data shown in Fig. 5A (Fig. 5B). Interestingly, cell cycle analysis showed significant differences in the number of GEP-shRNA-transfected Hep3B cells of 
A

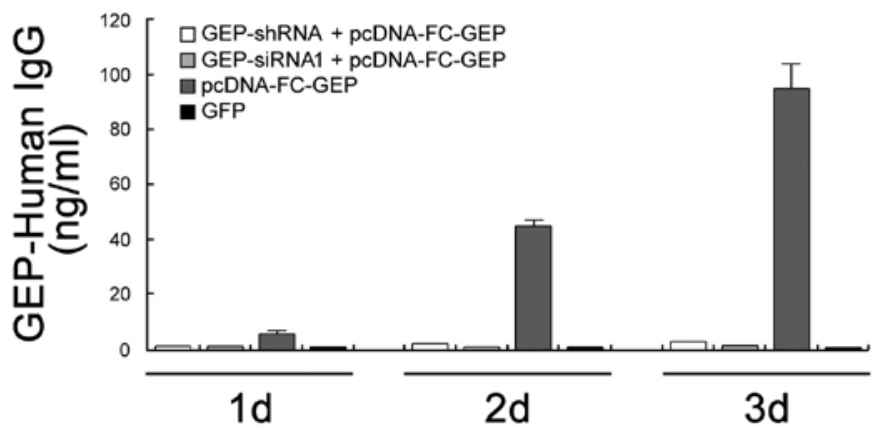

B

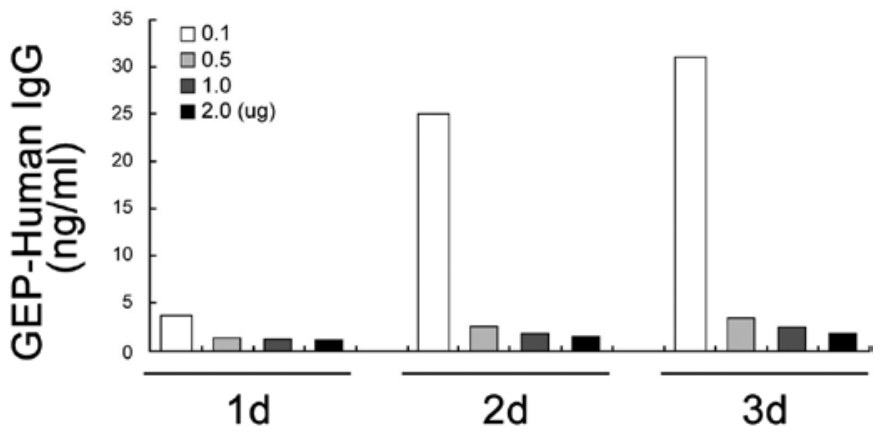

C

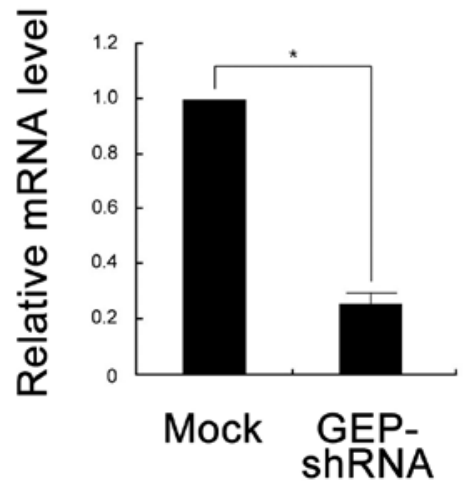

Figure 4. Synthesis of the GEP-shRNA containing GEP-siRNA1 sequences and its GEP-suppression effect in Hep3B cells. (A), GEP-shRNA and GEP-siRNA1 exhibited a similar GEP suppression effect in pcDNA-FC-GEP-transfected cells, as measured using ELISA. (B), The expression of GEP was suppressed in a dose-dependent manner by GEP-shRNA. (C), The analysis of the level of expression of the endogenous GEP mRNA in Hep3B cells transfected with GEP-shRNA using real-time RT-PCR revealed that this molecule was significantly decreased in Hep3B cells ( $(\mathrm{p}<0.05)$ after GEP-shRNA transfection. d, days after transfection.

their G0/G1, S, and G2/M phases compared with the control cells (Luciferase-shRNA-transfected Hep3B cells); namely, compared with the control group, significantly small number of GEP-shRNA-transfected cells were in the G0/G1 phase $(58.16 \pm 1.08 \%$ vs $69.54 \%)$ and significantly large number of GEP-shRNA-transfected cells were in the $S$ phase $(20.64 \pm 1.92 \%$ vs $15.67 \%)$ and in the $\mathrm{G} 2 / \mathrm{M}$ phase $(19.82 \pm 1.92 \%$ vs $14.25 \%)$ (Fig. 6A and B). These cell cycle data suggest that GEP-shRNA arrests the cell cycle at the G2/M phase.

Expression levels of cyclin D1 and $\alpha$-tubulin in Hep3B cells. The expression level of the cell-proliferative proteins, $\alpha$-tubulin and cyclin D1, was detected using Western blot analysis. As shown in Fig. 6C and D, the level of expression of these two proteins was significantly decreased in GEP-shRNA-transfected cells compared with the Luciferase-shRNA-transfected Hep3B cells. These results indicate that GEP-shRNA suppresses the expression of cell-proliferative proteins, thereby reducing the cell proliferation.
Regression of tumor volume by GEP-shRNA injection into tumor-bearing Balb/C nude mice. As shown in Fig. 7A, tumors continued to grow in control mice injected with LuciferaseshRNA and the maximum mass of excised tumors reached $1,373 \pm 250 \mathrm{~mm}^{3}$ at 31 days after injection. In contrast, the volume of the tumors in the GEP-shRNA-treated group was significantly smaller than that detected in the LuciferaseshRNA-treated group ( $\mathrm{p}<0.05)$ (Fig. 7B and C). Therefore, the down-regulation of GEP expression by GEP-shRNA can be a promising therapeutic treatment for HCC.

\section{Discussion}

HCC is a highly proliferative and vascularized tumor and its progress is closely related to angiogenesis and vascular remodeling (21). At present, a humanized anti-VEGF monoclonal antibody (22) and Sorafenib (23), a small tyrosine kinase inhibitor targeting VEGF receptors, are under consideration as efficient HCC chemotherapeutic agents, as they reduce angiogenesis, 
A

Hep3B

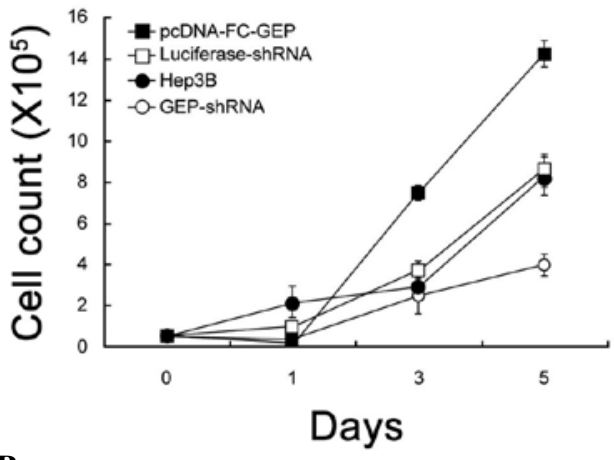

B

\section{Huh7}

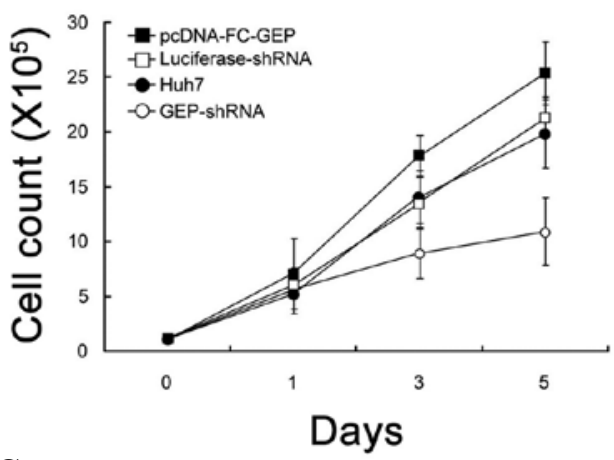

C

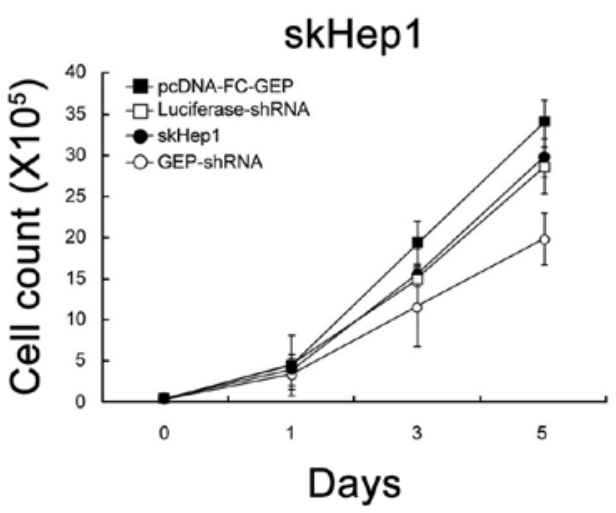

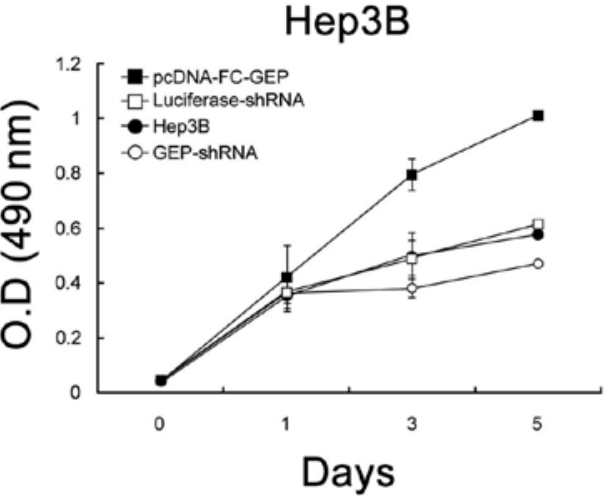

Huh7
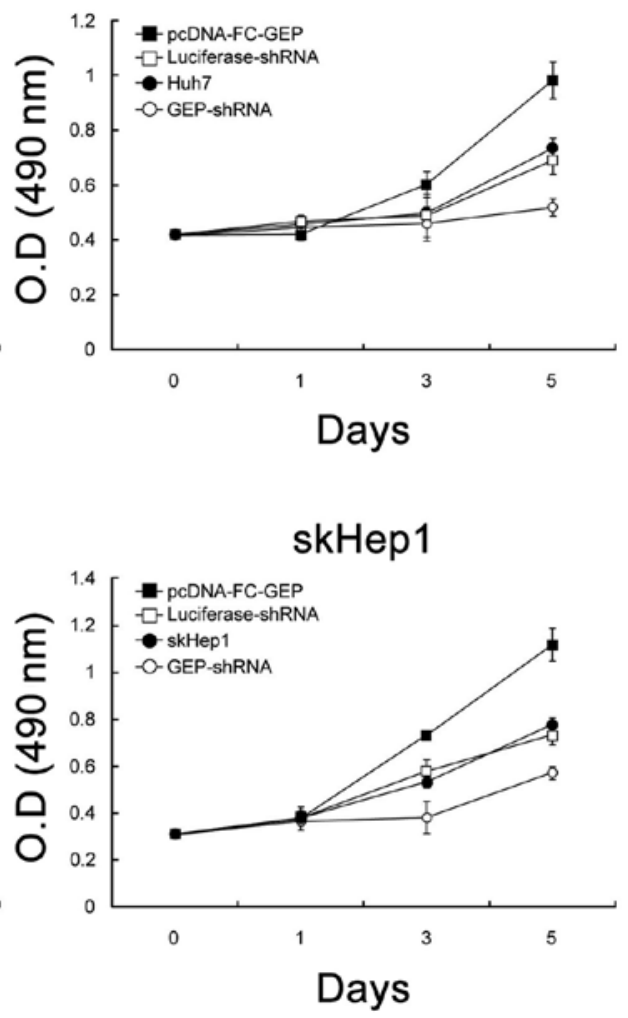

Figure 5. Suppression of GEP expression by GEP-shRNA led to a reduction of the proliferation and viability of HCC cell lines. (A), A cell-proliferation assay was performed at 1,3, and 5 days after transfection by counting cells using trypan blue staining. (B), Cell viability was measured at 1,3, and 5 days after GEP-shRNA transfection using an MTS assay (3-(4,5-dimethylthiazol-2-yl)-5-(3-carboxymethoxyphenyl)-2-(4-sulfophenyl)-2H-tetrazolium). All experiments were performed independently three times. pcDNA-FC-GEP was transfected as a positive control. An shRNA targeting the luciferase gene (Luciferase-shRNA) was used as a negative control. GEP-shRNA, HCC cells transfected with GEP-shRNA; Hep3B, Huh7, and skHep1, non-treated HCC cells used in mock experiments.

thus reducing cancer cell proliferation in HCC tumorigenesis. However, no significant increase in survival rate or therapeutic effect was demonstrated during those trials (24). Another study reported that Sorafenib improves the median survival time of patients diagnosed with advanced HCC by nearly 3 months (8); however, severe side effects such as significant risk of bleeding, were also reported (6). HCC is relatively resistant to systemic therapy (25). RNAi methods can be used to overcome this chemotherapeutic resistance, as they use a naturally occurring mechanism of sequence-specific gene-expression silencing (26). Furthermore, the novel finding of GEP overexpression in highly proliferative HCC tumor tissues provides a promising therapeutic target for HCC via the reduction of cell proliferation $(15,16)$. A growing number of studies dedicated to GEP report this molecule as a promising target molecule to cure HCC.

In this study, we examined the GEP-suppression effect of three different types of GEP-siRNAs and one shRNA. Specifically, the GEP-shRNA designed using the GEP-siRNA1 sequences yielded a decrease in the level of expression of the endogenous GEP mRNA and in tumor cell growth via the reduction of the cell-proliferative proteins $\alpha$-tubulin and cyclin D1 in GEP-shRNA-transfected Hep3B cells (Figs. 4-6). Moreover, GEP-shRNA yielded a significant regression of tumor volume in tumor-bearing Balb/C mice (Fig. 7). 
A

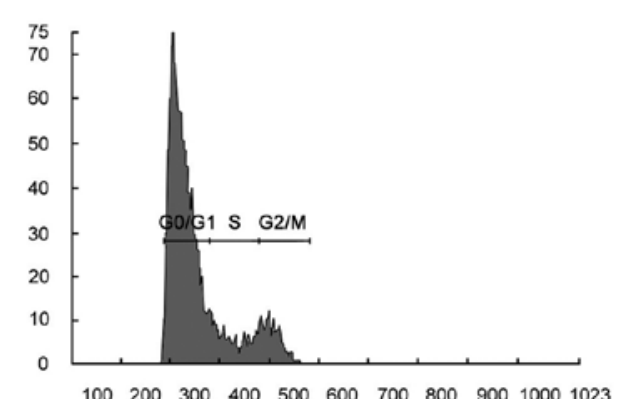

FL3 Lin

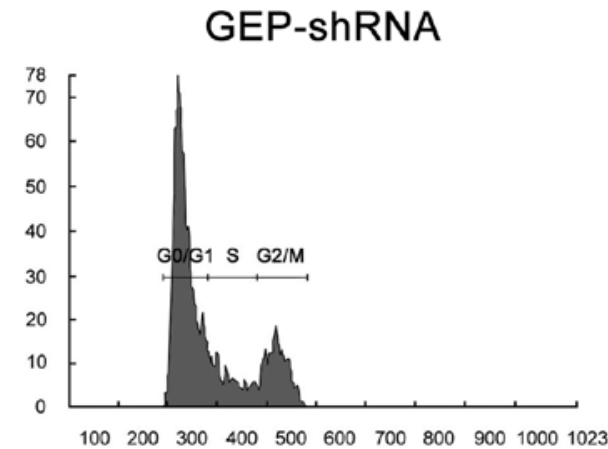

FL3 Lin

B

\begin{tabular}{c|ccc}
\hline & G0/G1 & S & G2/M \\
\hline Luciferase-shRNA & 69.54 & 15.67 & 14.25 \\
GEP-shRNA & $58.16 \pm 1.08$ & $20.64 \pm 1.98$ & $19.82 \pm 1.92$ \\
\hline
\end{tabular}

C

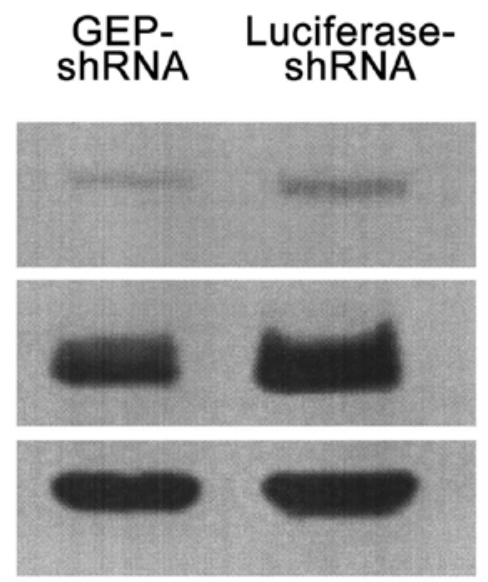

Cyclin D1

$\alpha$-tubulin

$\beta$-actin
D
Figure 6. GEP-shRNA induced cell-cycle arrest in Hep3B cells. (A and B), Cell-cycle analysis was performed using a flow cytometry with propidium iodide staining. The number of GEP-shRNA-transfected cells in the G0/G1 phase was decreased and in the $\mathrm{S}$ and $\mathrm{G} 2 / \mathrm{M}$ phases was increased compared with Luciferase-shRNA-transfected cells (used as mock). (C), The expression level of $\alpha$-tubulin and cyclin D1 was detected using Western blot analysis. (D), Relative amount of cyclin D1 and $\alpha$-tubulin, as assessed using the ImageJ program (obtained from the $\mathrm{NIH}$ ). All experiments were performed independently three times. ${ }^{* *} \mathrm{p}<0.01$.
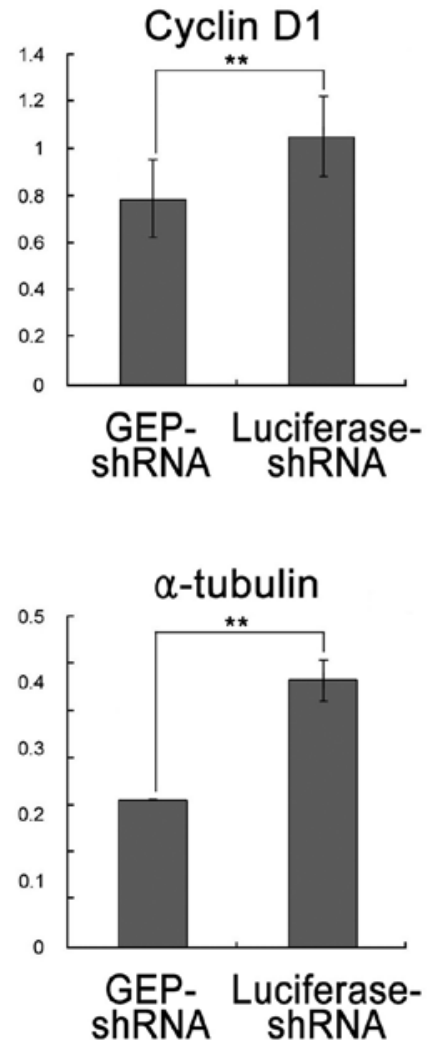

All GEP-siRNAs analyzed in this study yielded an inhibitory effect on GEP secretion, as assessed using an ELISA assay, because of the presence of tight stem-loop structures in all siRNA sequences (Fig. 3). A previous report showed that siRNA sequences located in stem-loop structures of RNA may be more functional during mRNA suppression (27). Our result (Fig. 3) showing that GEP-siRNA1 exhibited the strongest inhibitory effect on GEP secretion in Hep3B cells indicates that the GEP-siRNA1 oligonucleotide has a specific sequence that is more favorable for the blocking GEP expression. The GEP-shRNA designed using the sequence of GEP-siRNA1 yielded a similar blocking effect of GEP secretion (Fig. 4). GEP-shRNA transfection also demonstrated that GEP is positively associated with cell proliferation (Fig. 5), which is in accordance with the GEP-expression-blocking pattern reported by Cheung et al (15). Therefore, we concluded that the proliferation of HCC cells was markedly inhibited by GEP-shRNA. Furthermore, compared with previous results showing limited 
A

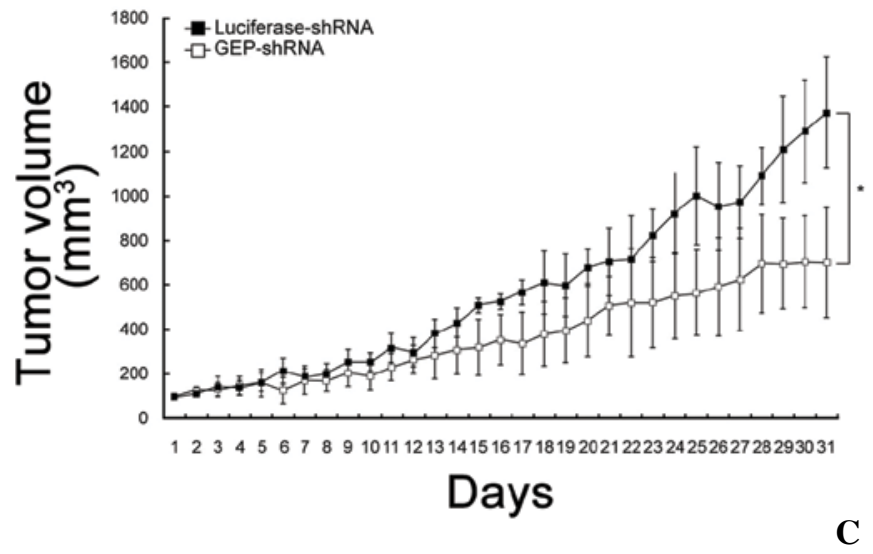

\section{GEP-shRNA}

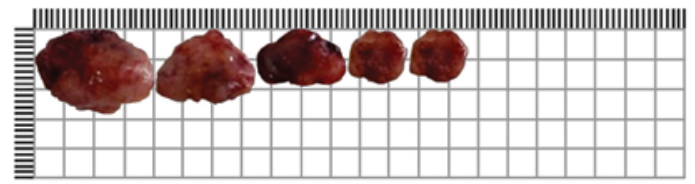

\section{Luciferase-shRNA}

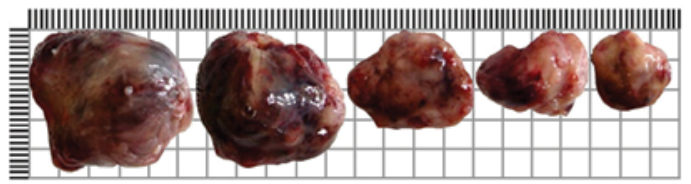

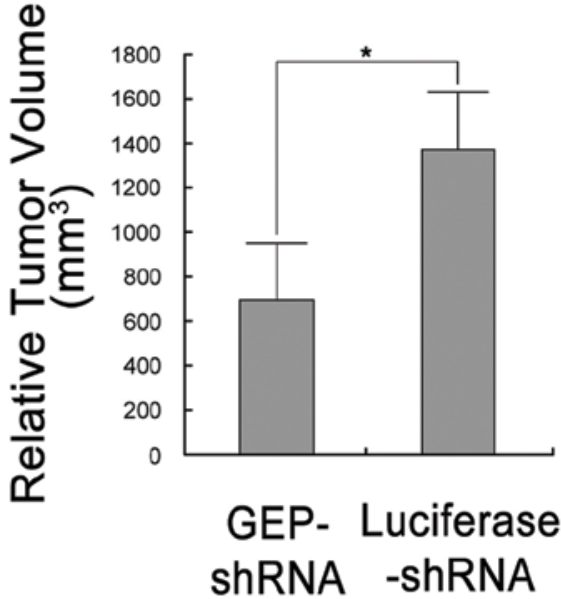

Figure 7. Tumor-suppressing effect of GEP-shRNA in a tumor-bearing mouse model. (A), The suppression of GEP expression by GEP-shRNA led to a decrease in tumor volume in a Hep3B xenograft mouse model for 31 days. Hep3B cells were inoculated subcutaneously into the femoral region. Tumor volume was measured using a digital caliper. The GEP-shRNA-injected group (five mice) showed a significant delay of tumor growth compared with the Luciferase-shRNA-injected group (five mice). (B), Tumors extracted at 31 days after transfection. (C), The relative tumor volume observed 31 days after GEP-shRNA transfection was significantly decreased compared with that of the Luciferase-shRNA-transfected group (used as mock; ${ }^{*} \mathrm{p}<0.05$ ).

regression in cell proliferation after the use of antisense GEP (15), the concentration of $0.5 \mu \mathrm{g}$ of GEP-shRNA can be considered as an intermediate-to-moderate level of treatment to achieve regression of cell proliferation (Fig. 4).

The cell cycle analysis led to the detection of a significant difference in the number of GEP-shRNA-transfected Hep3B cells in the G0/G1, G2/M, and S phases compared with control cells (Fig. 6B). The number of GEP-shRNA-transfected cells in the $\mathrm{G} 2 / \mathrm{M}$ and $\mathrm{S}$ phases was increased and in the $\mathrm{G} 0 / \mathrm{G} 1$ phase was decreased compared with the control group. We also investigated cell proliferation and cell cycle-related protein expression in Hep3B cells. Interestingly, we found that the $\alpha$-tubulin and cyclin D1 proteins were down-regulated in GEP-shRNAtransfected Hep3B cells (Fig. 6C and D). $\alpha$-tubulin plays a pivotal role in the $\mathrm{G} 2$ phase of the cell cycle (28). Consequently, the dysfunction or dysregulation of $\alpha$-tubulin protein can lead to a G2 cell-cycle arrest (29). Moreover, it was previously reported that cyclin D1 is a key regulator of cell proliferation (30). Therefore, the reduction of GEP could arrest the cell cycle via the down-regulation of cyclin D1 and $\alpha$-tubulin. Taken together, our data imply that the reduction of GEP may prevent HCC growth via tumor cell cycle arrest.

GEPoverexpression is closely associated with HCC development, e.g., in terms of large tumor size, which is related with a late stage of HCC (31). In this work, the tumorigenic effect of GEP was tested using a tumor-bearing athymic nude mouse model. We found that the treatment with GEP-shRNA led to a comparable reduction of tumor volume (1.9-fold decrease compared with that of observed in Luciferase-shRNA-treated mice; Fig. 7) after 31 days of GEP-shRNA treatment, which shows a similar antitumorigenic effect observed in mice treated with antisense GEP and anti-GEP monoclonal antibody for the management of HCC $(15,16)$.

However, still improvement of the GEP-shRNA is required for the clinical applications including the development of an effective delivery system of shRNA into tumors. To overcome this problem, we are developing a new viral vector system and cell permeable peptide sequences fused to the shRNA. We are in the process of studying a novel method to ameliorate the effectiveness of this delivery system.

In summary, this is the first report of targeting the GEP using RNAi (either siRNA or shRNA) for HCC treatment in association with a cancer cell-proliferation inhibitory effect. We concluded that GEP plays a pivotal role in hepatocarcinogenesis by clearly demonstrating that GEP-shRNA effectively inhibited tumor growth in vitro and in vivo via cell cycle arrest. Therefore, GEP-shRNA may be a safe and useful treatment for HCC therapy. 


\section{Acknowledgements}

This work was supported by the Catholic University of Korea Research Fund 2011, MKE and KOTEF through the Human Resource Training Project for Strategic Technology, GRRC of the Catholic University of Korea and the Biogreen21 program (PJ007186), Rural Development Administration, Republic of Korea. We appreciate the provision of CAPSID by Drs Eun Jung Jun and Heuiran Lee at the University of Ulsan, College of Medicine.

\section{References}

1. Thomas MB, Jaffe D, Choti MM, Belghiti J, Curley S, Fong Y, Gores G, Kerlan R, Merle P, O'Neil B, Poon R, Schwartz L, Tepper J, Yao F, Haller D, Mooney M and Venook A: Hepatocellular carcinoma: consensus recommendations of the National Cancer Institute Clinical Trials Planning Meeting. J Clin Oncol 28: 3994-4005, 2010.

2. Llovet JM, Burroughs A and Bruix J: Hepatocellular carcinoma. Lancet 362: 1907-1917, 2003.

3. El-Serag HB and Mason AC: Rising incidence of hepatocellular carcinoma in the United States. N Eng J Med 340: 745-750, 1999.

4. Benvegnù L, Gios M, Boccato S and Alberti A: Natural history of compensated viral cirrhosis: a prospective study on the incidence and hierarchy of major complications. Gut 53: 744-749, 2004

5. Sangiovanni A,Prati GM, Fasani P, Ronchi G, Romeo R, Manini M, Del Ninno E, Morabito A and Colombo M: The natural history of compensated cirrhosis due to hepatitis $\mathrm{C}$ virus: a 17-year cohort study of 214 patients. Hepatology 43: 1303-1310, 2006.

6. El-Serag HB and Rudolph KL: Hepatocellular carcinoma: epidemiology and molecular carcinogenesis. Gastroenterology 132 2557-2576, 2007.

7. Cabrera R and Nelson DR: The management of hepatocellular carcinoma. Aliment Pharmacol Ther 31: 461-476, 2010.

8. Altekruse SF, McGlynn KA and Reichman ME: Hepatocellular carcinoma incidence, mortality, and survival trends in the United States from 1975 to 2005. J Clin Oncol 27: 1485-1491, 2009.

9. Castello G, Costantini S and Scala S: Targeting the inflammation in $\mathrm{HCV}$-associated hepatocellular carcinoma: a role in the prevention and treatment. J Transl Med 8: 109-119, 2010.

10. Chen X, Cheung ST, So S, Fan ST, Barry C, Higgins J, Lai KM, Ji J, Dudoit S, Ng IO, van de Rijn M, Botstein D and Brown PO Gene expression patterns in human liver cancers. Mol Biol Cell 13: 1929-1939, 2002.

11. Creppy EE, Traoré A, Baudrimont I, Cascante M and Carratú MR: Recent advances in the study of epigenetic effects induced by the phycotoxin okadaic acid. Toxicology 181-182: 43-47, 2002.

12. Shoyab M, McDonald VL, Byles C, Todaro GJ and Plowman GD Epithelins 1 and 2: isolation and characterization of two cysteinerich growth-modulating proteins. Proc Natl Acad Sci USA 87: 7912-7916, 1990

13. Hrabal R, Chen Z, James S, Bennett HP and Ni F: The hairpin stack fold, a novel protein architecture for a new family of protein growth factors. Nat Struct Biol 3: 747-752, 1996

14. He Z, Ong CH, Halper J and Bateman A: Progranulin is a mediator of the wound response. Nat Med 9: 225-229, 2003

15. Cheung ST, Wong SY, Leung KL, Chen X, So S, Ng IO and Fan ST: Granulin-epithelin precursor overexpression promotes growth and invasion of hepatocellular carcinoma. Clin Cancer Res 10: 7629-7636, 2004.
16. Ho JC, Ip YC, Cheung ST, Lee YT, Chan KF, Wong SY and Fan ST: Granulin-epithelin precursor as a therapeutic target for hepatocellular carcinoma. Hepatology 47: 1524-1532, 2008.

17. Huang C, Li M, Chen C and Yao Q: Small interfering RNA therapy in cancer: mechanism, potential targets, and clinical applications. Expert Opin Ther Targets 12: 637-645, 2008.

18. Castanotto D and Rossi JJ: The promises and pitfalls of RNAinterference-based therapeutics. Nature 457: 426-433, 2009.

19. Yan X, Chua MS, He J and So SK: Small interfering RNA targeting CDC25B inhibits liver tumor growth in vitro and in vivo. Mol Cancer 7: 19-27, 2008.

20. Fu Y, Huang J, Wang KS, Zhang X and Han ZG: RNA interference targeting CITRON can significantly inhibit the proliferation of hepatocellular carcinoma cells. Mol Biol Rep 38: 693-702, 2011.

21. Fernández M, Semela D, Bruix J, Colle I, Pinzani M and Bosch J: Angiogenesis in liver disease. J Hepatol 50: 604-620, 2009.

22. Siegel AB, Cohen EI, Ocean A, Lehrer D, Goldenberg A, Knox JJ, Chen H, Clark-Garvey S, Weinberg A, Mandeli J, Christos P, Mazumdar M, Popa E, Brown RS Jr, Rafii S and Schwartz JD: Phase II trial evaluating the clinical and biologic effects of bevacizumab in unresectable hepatocellular carcinoma. J Clin Oncol 26: 2992-2998, 2008.

23. Llovet JM, Ricci S, Mazzaferro V, Hilgard P, Gane E, Blanc JF, De Oliveira AC, Santoro A, Raoul JL, Forner A, Schwartz M, Porta C, Zeuzem S, Bolondi L, Greten TF, Galle PR, Seitz JF, Borbath I, Häussinger D, Giannaris T, Shan M, Moscovici M, Voliotis D and Bruix J: SHARP Investigators Study Group. Sorafenib in advanced hepatocellular carcinoma. N Engl J Med 359: 378-390, 2008.

24. Bergé M, Bonnin P, Sulpice E, Vilar J, Allanic D, Silvestre JS, Lévy BI, Tucker GC, Tobelem G and Merkulova-Rainon T: Small interfering RNAs induce target-independent inhibition of tumor growth and vasculature remodeling in a mouse model of hepatocellular carcinoma. Am J Pathol 177: 3192-3201, 2010.

25. Yau T, Chan P, Epstein R and Poon RT: Evolution of systemic therapy of advanced hepatocellular carcinoma. World J Gastroenterol 14: 6437-6441, 2008

26. Wu Z, Li X, Zeng Y, Zhuang X, Shen H, Zhu H, Liu H and Xiao H: In vitro and in vivo inhibition of MRP gene expression and reversal of multidrug resistance by siRNA. Basic Clin Pharmacol Toxicol 108: 177-184, 2011.

27. Yoshinari K, Miyagishi M and Taira K: Effects on RNAi of the tight structure, sequence and position of the targeted region. Nucleic Acids Res 32: 691-699, 2004.

28. Rusan NM, Fagerstrom CJ, Yvon AM and Wadsworth P: Cell cycle-dependent changes in microtubule dynamics in living cells expressing green fluorescent protein-alpha tubulin. Mol Biol Cell 12: 971-980, 2001

29. Guo WH, Yuan LH, Xiao ZH, Liu D and Zhang JX: Overexpression of SUMO-1 in hepatocellular carcinoma: a latent target for diagnosis and therapy of hepatoma. J Cancer Res Clin Oncol 137: 533-541, 2011

30. Witzel II, Koh LF and Perkins ND: Regulation of cyclin D1 gene expression. Biochem Soc Trans 38: 217-222, 2010.

31. Hirohashi S, Bulm HE, Ishak KG, et al: Hepatocellular carcinoma. In: World Health Organization Classification of Tumors. Pathology and Genetics Tumors of the Digestive System. Hamilton SR and Aaltonen LA (eds). World Health Organization, Geneva, pp158-172, 2000. 